\title{
The Fg12/fibroleukin prothrombinase contributes to immunologically mediated thrombosis in experimental and human viral hepatitis
}

\author{
Philip A. Marsden, ${ }^{1,2}$ Qin Ning, ${ }^{3}$ Laisum S. Fung, ${ }^{4}$ Xioping Luo, ${ }^{3}$ Yue Chen, ${ }^{3}$ \\ Michael Mendicino, ${ }^{4}$ Anand Ghanekar, ${ }^{4}$ Jeremy A. Scott, ${ }^{2}$ Teresa Miller, ${ }^{4}$ \\ Camie W.Y. Chan, ${ }^{4}$ Mathew W.C. Chan, ${ }^{4}$ Wei He, ${ }^{4}$ Reginald M. Gorczynski,,${ }^{1,4}$ \\ David R. Grant, ${ }^{4}$ David A. Clark, ${ }^{1,4,5}$ M. James Phillips, ${ }^{1,4,6}$ and Gary A. Levy ${ }^{1,4}$
}

${ }^{1}$ CIHR Group on Cellular and Molecular Mechanisms of Organ Injury, Toronto, Ontario, Canada

${ }^{2}$ St. Michael's Hospital and Department of Medicine, University of Toronto, Toronto, Ontario, Canada

${ }^{3}$ Department of Infectious Disease, Tongji Hospital of Tongji Medical College, Huazhong University

of Science and Technology, Wuhan, People's Republic of China

${ }^{4}$ Multi Organ Transplant Program, University Health Network, University of Toronto, Toronto, Ontario, Canada

${ }^{5}$ McMaster University Medical Center, Hamilton Health Sciences Corp., Hamilton, Ontario, Canada

${ }^{6}$ Department of Pathology, Hospital for Sick Children, Toronto, Ontario, Canada

\begin{abstract}
Fibrin deposition and thrombosis within the microvasculature is now appreciated to play a pivotal role in the hepatocellular injury observed in experimental and human viral hepatitis. Importantly, the pathways by which fibrin generation is elicited in viral hepatitis may be mechanistically distinct from the classical pathways of coagulation induced by mechanical trauma or bacterial lipopolysaccharide (LPS). In the setting of murine hepatitis virus strain-3 (MHV-3) infection, a member of the Coronaviridae, activated endothelial cells and macrophages express distinct cell-surface procoagulants, including a novel prothrombinase, Fgl2/fibroleukin, which are important for both the initiation and localization of fibrin deposition. To assess the role of Fgl2/fibroleukin in murine viral hepatitis we generated a Fgl2/fibroleukin-deficient mouse. Peritoneal macrophages isolated from Fgl2/fibrolenkin ${ }^{-/-}$mice did not generate a procoagulant response when infected with MHV-3. Fibrin deposition and liver necrosis were markedly reduced, and survival was increased in mice infected with MHV-3. To address the relevance of Fgl2/fibroleukin in human chronic viral hepatitis we studied patients with minimal and marked chronic hepatitis B. We detected robust expression of Fgl2/fibroleukin mRNA transcripts and protein in liver tissue isolated from patients with marked chronic hepatitis B. Fibrin deposition was strongly associated with Fgl2/fibroleukin expression. Collectively, these data indicate a critical role for Fgl2/fibroleukin in the pathophysiology of experimental and human viral hepatitis.
\end{abstract}

J. Clin. Invest. 112:58-66 (2003). doi:10.1172/JCI200318114.

\section{Introduction}

Many of the viral pathogens known to cause liver disease are not directly cytopathic for the hepatocyte. For instance, the acute and chronic liver diseases caused by the hepatitis B virus reflect hepatocellular injury induced by an immune response to the virus rather than direct viral-induced hepatocellular necrosis. For

Received for publication February 12, 2003, and accepted in revised form April 18, 2003.

Address correspondence to: Philip A. Marsden, University of Toronto, Room 7358, Medical Sciences Building, 1 King's College Circle, Toronto, Ontario M5S 1A8, Canada. Phone: (416) 978-2441; Fax: (416) 978-8765;

E-mail: p.marsden@utoronto.ca.

Philip A. Marsden and Qin Ning contributed equally to this work. Conflict of interest: The authors have declared that no conflict of interest exists.

Nonstandard abbreviations used: tissue factor (TF); murine hepatitis virus type 3 (MHV-3); postcoitus (pc); alanine aminotransferase (ALT); hepatitis B surface antigen (HBsAg); hepatitis B e-antigen ( $\mathrm{HBeAg})$; hepatitis B viral capsid ( $\mathrm{HBcAg}$ ); severe acute respiratory syndrome (SARS). example, IFN- $\gamma$ derived from activated $\mathrm{T}$ cells within the liver figures prominently in viral-induced liver disease in humans as well as in animal models (1). Another especially important effector phase in the immune response to viral infection of the liver is fibrin deposition and thrombosis within the microvasculature (2). This is an important concept that offers hope for the development of novel treatment approaches to viralinduced liver disease. Herein we provide new insight into the importance of novel immune procoagulants in the pathogenesis of viral hepatitis.

Tissue factor (TF), the transmembrane receptor for factor VII, is the major procoagulant for the classical pathway and is known to initiate the extrinsic pathway of coagulation in response to LPS $(3,4)$. In the presence of calcium, the TF/factor VIIa complex activates the zymogen precursor factor $\mathrm{X}$. Factor Xa is a serine protease that is the classically known physiological activator of prothrombin. Efficient thrombin generation by factor $\mathrm{Xa}$ requires calcium, an electronegative phospholipid surface, and the nonenzymatic cofactor, factor Va. 
Current evidence indicates that the pathways by which vascular thromboses are elicited in viral hepatitis are mechanistically distinct from the classical pathways of coagulation induced by mechanical trauma or bacterial lipopolysaccharide (LPS). The in vitro induction of a procoagulant response in mononuclear cells by murine hepatitis virus type-3 (MHV-3) correlates with the disease susceptibility in strains of mice (5). MHV-3 is a member of the Coronaviridae, a group of positive-stranded, enveloped RNA viruses. Peritoneal macrophages from BALB/c or C57Bl/6 mice, strains in which MHV-3 infection results in fatal acute fulminant hepatitis, respond to the virus with a robust procoagulant response. In contrast, peritoneal macrophages from A/J mice, a strain fully resistant to MHV-3, generate no increase in procoagulant above control levels. This procoagulant response represented a functional marker for susceptibility to MHV-3 infection in inbred strains of mice, suggesting that this response is important in the pathogenesis of coronavirus MHV-3-induced disease. Activated endothelial cells and macrophages express distinct cell-surface procoagulants important for both the initiation and localization of fibrin deposition in viral-induced liver disease. The cloning and characterization of a novel procoagulant induced by MHV-3 infection of murine macrophages has been reported (6). Fgl2/fibroleukin is an immune coagulant with the ability to directly cleave prothrombin to thrombin (6-10). Initially cloned from $\mathrm{CD}^{+}$cytotoxic $\mathrm{T}$ cells, $\mathrm{Fgl}$ /fibroleukin protein shares homology of its carboxyl terminus with fibrinogen $\beta$ and $\gamma$ chains (11). Fgl2/fibroleukin protein generated in a baculovirus expression system exhibits no measurable prothrombinase activity until reconstituted into negatively charged phospholipid vesicles (e.g., containing phosphatidyl-L-serine) (10). As with factor Xa, catalytic activity is dependent on calcium and is enhanced by factor Va. Site-directed mutagenesis of Ser89 of the murine protein indicated that Fgl2/fibroleukin has the characteristics of a serine protease and that Ser89 was critical for the prothrombinase activity of $f g l 2$. In contrast to factor $\mathrm{Xa}$, the $\mathrm{Fgl} 2 /$ fibroleukin prothrombinase activity is not inhibited by antithrombin III.

The human and murine genes for the Fgl2/fibroleukin prothrombinase have been cloned and characterized and localized to chromosomes 7 and 5 , respectively $(8,12)$. Of note, expression of the Fgl2/fibroleukin prothrombinase mRNA is subject to transcriptional regulation, exhibiting both constitutive and inducible expression. For example, steady-state mRNA expression of Fgl2/fibroleukin prothrombinase is markedly upregulated by IFN- $\gamma(13,14)$. In vivo studies indicate robust expression of the Fgl2/fibroleukin prothrombinase in hepatic macrophages and endothelial cells in MHV-3 murine hepatitis models and patients with fulminant hepatic failure $(7,15)$.

Given that the Fgl2/fibroleukin may be important for both the initiation and localization of fibrin deposition in viral hepatitis, we generated an Fgl2/fibroleukin-defi- cient mouse. Importantly, we found that fibrin deposition and liver necrosis were markedly reduced and survival was increased in mice infected with the MHV-3 coronavirus. The relevance of these findings to patients with virus-induced liver disease is underscored by the prominent expression of $\mathrm{Fgl}$ /fibroleukin prothrombinase mRNA transcripts and immunoreactive protein, and strong association with fibrin deposition, in liver tissue from patients with marked chronic hepatitis $\mathrm{B}$-induced disease.

\section{Methods}

All animal experiments in the study were carried out according to the guidelines of the Canadian Council on Animal Care and approved by the University of Toronto and/or University Health Network Committees on Animal Experimentation. Informed consent was obtained from human study participants, and the research protocol was reviewed and approved by the institutional review board of Tonji Hospital, Wuhan, People's Republic of China.

Generation of Fgl2/fibroleukin prothrombinase-deficient mice. The targeting construct was derived from a mouse $129 \mathrm{~Sv} / \mathrm{J}$ strain genomic library and transfected into $129 \mathrm{~Sv} / \mathrm{J}$ ES cells by electroporation. Appropriately targeted ES clones were injected into $\mathrm{C} 57 \mathrm{Bl} / 6$ blastocysts. Chimeric males were mated with $\mathrm{C} 57 \mathrm{Bl} / 6$ females. Mice were interbred from successive generations of $\mathrm{Fgl2}_{\text {fibroleukin }}{ }^{+-}$founders, and thus littermates represent a mixed $129 \mathrm{~Sv} / \mathrm{J} \times \mathrm{C} 57 \mathrm{Bl} / 6$ genetic background. Southern blot analysis and multiplex PCR were used to genotype animals (details of probes and oligonucleotides are available from the authors upon request). The $129 \mathrm{~Sv} / \mathrm{J}$ inbred strain and the $\mathrm{C} 57 \mathrm{Bl} / 6$ inbred strain are both MHV-3 sensitive (16). To assess the effects of strain background on loss of $\mathrm{Fgl}_{2} /$ fibrolenkin $^{-/-}$ homozygotes and response to $\mathrm{MHV}-3$ infection, Fgl2/fibroleukin ${ }^{+/-}$mice were backcrossed with C57Bl/6 inbred mice for ten successive generations. The fidelity of the backcross was assessed by genotyping $\mathrm{Fgl2} /$ fibroleukin $^{+/-}$mice derived from the tenth generation for two informative DNA markers per chromosome (i.e., different alleles in $129 \mathrm{~Sv} / \mathrm{J}$ versus C57Bl/6). Unless stated otherwise, all studies were performed on the mixed $129 \mathrm{~Sv} / \mathrm{J} \times \mathrm{C} 57 \mathrm{Bl} / 6$ genetic background.

Embryo studies. Uteri were removed from euthanized pregnant mice on postcoitum (pc) days 7.5-11.5, were fixed in freshly prepared buffered $4 \%$ paraformaldehyde for 18 hours, and then embedded in paraffin. Serial sections were cut and fixed to Aptex-coated slides. Every tenth slide was stained with hematoxylin and eosin. Adjacent sections were stained with polyclonal rabbit anti-fgl2 at 1/10,000 dilution or control rabbit IgG 1.25 $\mu \mathrm{g} / \mathrm{ml}$ as described (17), without microwave treatment.

Hematological studies in mice. Mice were anesthetized, followed by percutaneous cardiac puncture for blood aspiration. Approximately $500 \mu \mathrm{l}$ of blood was collected into a Microtainer tube containing EDTA (Becton Dickinson Corp., Franklin Lakes, New Jersey, USA). 
Hemoglobin level, platelet count, and leukocyte count were performed by the University Health Network Medical Laboratories (Toronto, Ontario, Canada), where the automated equipment was readjusted to standardize for mouse samples. The Hemochron Jr. II Microcoagulation System (International Technidyne Corp., Edison, New Jersey, USA) was used for immediate measurement of prothrombin time and activated partial thromboplastin times. Bleeding times were measured as described (18).

Procoagulant activity and Fgl2 expression in mice. Thioglycollate-elicited macrophages $\left(4 \times 10^{6} / \mathrm{ml}\right)$ were exposed in vitro to MHV-3 (MOI 1), $10 \mu \mathrm{g} / \mathrm{ml}$ LPS (Eschericia coli serotype 0111; Sigma-Aldrich, St. Louis, Missouri, USA), or vehicle for 8 hours for procoagulant activity measurements. After three cycles of freezing, thawing, and sonication, extracts were assayed for the capacity to shorten the spontaneous clotting time of normal citrated human plasma in a one-stage clotting assay, as described previously (10). The time for appearance of a fibrin gel was recorded. First-strand cDNA was synthesized using the SuperScript Preamplification protocol (Life Technologies Inc., Rockville, Maryland, USA) followed by RT-PCR amplification using a set of $f g l 2$-specific primers spanning exon 1 and 2 (sense: $5^{\prime}$-TGC TCA AAG AAG TGC GGA CCC T-3'; antisense: $5^{\prime}$-GCC CCA CTG CTT CTC CTT CCT-3'; 430 bp). RT-PCR was also performed in the in vivo setting using tissues harvested from mice injected intraperitoneally with 1,000 PFU of MHV-3. Total cellular RNA was isolated 16 hours after infection, and RT-PCR was performed as described above.

Susceptibility to MHV-3 infection and LPS exposure. For LPS studies, mice ( $n=10$ per group, age 3 to 6 months) received LPS (30 mg/ $\mathrm{kg}$ E. coli serotype O55:B5, intraperitoneally) as well as buprenorphine and saline for analgesia and fluid resuscitation $(0.1 \mathrm{mg} / \mathrm{kg}$ in $2 \mathrm{ml}$ saline, subcutaneously at $t=0$ and 12 hours), respectively. Mice were monitored every 2 hours for 24 hours for morbidity and mortality. Any animals judged to be suffering were euthanized by cervical dislocation. $\mathrm{C} 57 \mathrm{Bl} / 6$ and $\mathrm{C} 3 \mathrm{H} / \mathrm{HeJ}$ strains were used as LPS-sensitive and LPShyporesponsive mouse controls, respectively. For MHV-3 studies, mice ( $n=10$ per group, age 3 to 6 months) were infected via intraperitoneal injection with $1,000 \mathrm{PFU}$ of MHV-3. Multiple independent experiments were performed using different preparations of viral stocks. Following infection, mice were housed in presterilized cages in an isolated environment and given food and water ad libitum. Mortality was scored when animals become moribund. Livers were removed from all euthanized mice and analyzed for viral titers, liver histology, and fibrin deposition. Frozen liver tissue was homogenized and viral titers determined on monolayers of L2 cells in a standard plaque assay (19). For histological examination and quantification of necrosis and fibrin deposition, liver tissue was fixed in $10 \%$ buffered formalin for 4 hours followed by routine tissue processing and staining using an automated tissue processor, following which the tissue blocks were embedded in paraffin. Livers were then sectioned $5-\mu \mathrm{m}$ thick and stained with hematoxylin and eosin and with martius/scarlet/blue. Liver sections were immunostained for fibrin using the Nexes immunostaining method, an automated avidin-biotin method using protease 1 treatment (Ventana Medical Systems, Tuscon, Arizona, USA) in which a purified $\mathrm{Ab}$ (a specific biotinylated 1:100 diluted anti-rabbit IgG; Vector Laboratories, Burlingame, California, USA) was used. Sections were scored in a blinded fashion to determine the extent of necrosis and fibrin deposition. The surface area of the liver examined was equal in all instances and corresponded to ten contiguous histological fields at low magnification $(\times 100)$. Sera from all animals were analyzed quantitatively for alanine aminotransferase (ALT) using a Worthington Statzyme GTP kit (Worthington Biochemical Corp., Lakewood, New Jersey, USA) as described previously (19).

Studies in patients with viral hepatitis $B$. Patients were recruited at Tonji Hospital. Biochemical, histological, and clinical features were used to define patients with chronic viral hepatitis B (marked chronic viral hepatitis B versus minimal chronic viral hepatitis B). Diagnostic criteria and laboratory investigations used standard definitions (20). The evaluation of patients with chronic viral hepatitis B infection included a thorough history and physical examination, with special emphasis on risk factors for coinfection (with hepatitis C, D, or HIV), alcohol use, and family history of viral hepatitis B infection and liver cancer. Percutaneous liver biopsies were obtained for the purposes of guiding treatment decisions (21). Hepatitis B viral markers and serology (hepatitis B surface antigen [HBsAg], hepatitis B e-antigen [HBeAg], anti-hepatitis B viral capsid [anti-HBcAg]) were assessed using ELISA assay kits obtained from Shanghai Shi yue Ke Hua Biological Science Inc. (Shanghai, People's Republic of China) and/or Abbott Laboratories (Chicago, Illinois, USA) according to the manufacturer's instructions. Hepatitis B viral DNA loads were assessed by PCR (sense: $5^{\prime}$ TTT GGG GCA TGG ACA TTG AC-3'; antisense: $5^{\prime}$-CCC ACC TTA TGA GTC CAA GG-3'). Twenty-three patients with marked chronic hepatitis $B$ and 13 patients with minimal chronic viral hepatitis B were evaluated. Sections of liver tissue were scored in a blinded fashion as to the histological diagnosis, fibrin deposition, and Fgl2/fibroleukin immunostaining. For fibrin detection, a rabbit-anti-human fibrinogen $\mathrm{Ab}$ (DAKO Corp., Carpinteria, California, USA) known to react with fibrinogen and fibrin in human tissues (7) was used. A CD68 Ab (DAKO Corp.) was used to detect macrophages (Kupffer cells). For Fgl2/fibroleukin immunostaining, a polyclonal rabbit $\mathrm{Ab}$ was used as described (17). Immunoperoxidase staining (dark brown) used the avidin-biotin complex method. For the in situ hybridization studies, a 169-bp DNA fragment from the carboxyl end of the human Fgl2/fibroleukin coding region was used as a template to synthesize anti-sense digoxigenin-11-UTP-labeled 
a

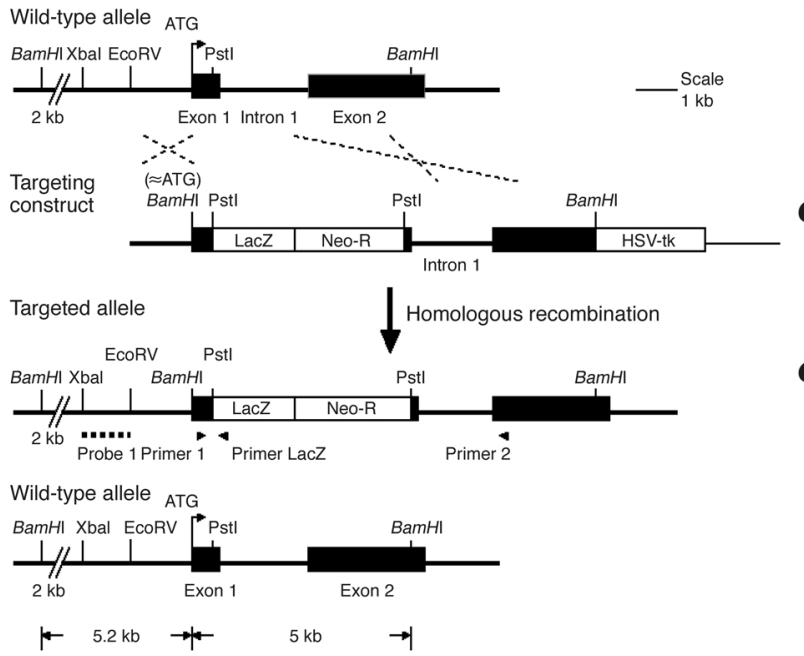

b

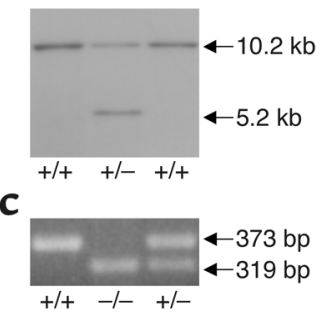

d

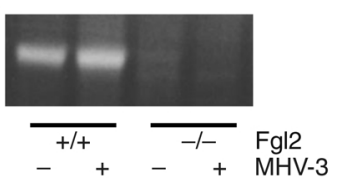

Figure 1

Generation of Fgl2/fibroleukin-deficient mice. (a) Targeting strategy for fg/2 gene. The LacZ reporter and PGK-neo gene were inserted within the first of two coding exons, and the Fgl2/fibroleukin initiator methionine codon in exon 1 was mutated to a $\mathrm{BamHl}$ site. Arrowheads represent primers used for multiplex PCR genotyping. The probe used for genomic Southern blot analysis is indicated by the dotted line. (b) Genomic Southern blot analysis of ES cell clones. The 10.2-kb or 5.2-kb BamHI restriction fragments are evident in wild-type or knockout alleles, respectively. (c) Multiplex PCR for genotyping mice amplified a 319-bp amplicon from the knockout allele or a 373-bp amplicon from the wild-type allele. (d) RT-PCR analysis of MHV3-treated macrophages ( 8 hours). Neither constitutive nor induced mRNA was detected in the null knockout mice.

cRNA probes (Hoffman-La Roche AG, Basel, Switzerland) and detected using the alkaline phosphatase method, as described (7).

Statistical analyses. All data are reported as the mean value plus or minus SEM, with sample sizes stated throughout. Mendelian heritability and survival curves were statistically assessed using the Chi-squared test with a Fisher exact test and Kaplan-Meier survival analysis, respectively (StatView V4.5; Abacus Concepts Inc., San Francisco, California, USA). $P$ values less than 0.05 were considered significant.

\section{Results}

Generation of Fgl2/fibrolenkin prothrombinase-deficient mice. To investigate the in vivo role(s) for Fgl2/fibroleukin in leukin ${ }^{+/}$, Fgl2/fibroleukin ${ }^{+/}$, and Fgl2/fibroleukin ${ }^{-/-}$mice, respectively (observed ratio 1:1.7:0.6 versus expected $1: 2: 1$ ratio; $\left.\chi^{2}=20.6 ; P<0.001\right)$. Importantly, $40 \%$ of Fgl2/fibrolenkin ${ }^{-1-}$ homozygotes were lost. At pe day 10.5 , genotyping of embryos indicated that an important degree of embryo loss was occurring early in development. To determine when embryo losses occurred, zygotes and uterine tissue were examined by light microscopy and immunohistochemistry using a polyclonal anti-Fgl2 Ab from pc days 7.5-11.5. On pc day 7.5 , embryos and uterine decidua appeared normal. Starting at pc day 8.5, many of the Fgl2/fibroleukin ${ }^{-/}$ embryos showed moderate to severe hemorrhage at implantation sites, with extravasation of maternal erythrocytes between the mural trophectoderm and

\section{Figure 2}

Fgl2/fibroleukin-deficient mice have hemorrhage at the feto-maternal interface. Zygotes and uterine tissue were examined by light microscopy and immunohistochemistry using a polyclonal anti-Fgl2 Ab. Feto-maternal interface of $\mathrm{Fgl} / 2 /$ fibroleukin $^{+/+}\left(\mathrm{Fgl}-2^{+/+}\right)$zygotes at pc day 8.5 , as assessed by hematoxylin and eosin staining (a) and $\mathrm{Fgl} 2$ immunostaining (b). Murine trophectoderm is positive for $\mathrm{Fgl} 2$ immunostaining. Fg/2/fibroleukin ${ }^{-/}\left(\mathrm{Fgl}-2^{-/-}\right)$zygotes showed moderate to severe hemorrhage at implantation sites, with extravasation of maternal erythrocytes ( $r b c$; arrowhead) between the mural trophectoderm and Reichert's membrane (arrow) at the antimesometrial pole as assessed by hematoxylin and eosin staining (c) and negative Fgl2 immunostaining (d) (see Methods).
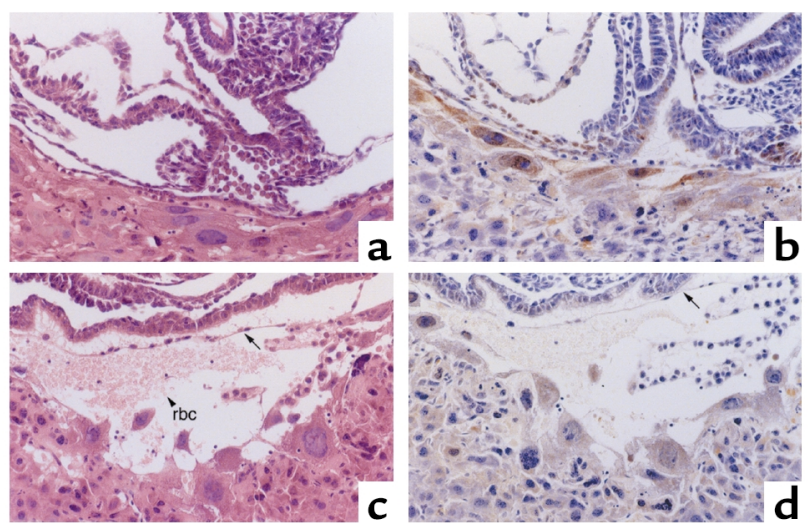
Table 1

Plasma clotting time (in seconds) by macrophages harvested from $\mathrm{Fg} / 2^{+/+}, \mathrm{Fg} / 2^{+/-}$, or $\mathrm{Fg} / 2^{-/-}$mice stimulated with $\mathrm{MHV}-3$ or LPS

\begin{tabular}{lccc} 
Stimulus & \multicolumn{3}{c}{ Fgl2/fibroleukin genotype } \\
& $+/+$ & $+/-$ & $-/-$ \\
None & $\geq 120$ & $\geq 120$ & $\geq 120$ \\
MHV-3 $(1,000 \mathrm{PFU})$ & $34 \pm 3$ & $41 \pm 2$ & $\geq 120$ \\
LPS $(10 \mu \mathrm{g})$ & $36 \pm 7$ & $38 \pm 5$ & $35 \pm 4$
\end{tabular}

Reichert's membrane, especially at the antimesometrial pole (Figure 2). Immunohistochemistry revealed that embryos with hemorrhages were Fgl2/fibroleukin deficient in mural trophectoderm. This negative staining contrasted with positive immunostaining in embryos that did not exhibit hemorrhages and the maternal decidua of Fgl2/fibrolenkin ${ }^{+/-}$mothers (Figure 2). Embryonic or maternal hemorrhage was otherwise not observed early or late in pregnancy. The loss of Fgl2/fibrolenkin ${ }^{-1-}$ homozygotes was not modified significantly on the C57Bl/ 6 inbred background. When Fgl2/fibrolenkin ${ }^{+/-}$mice were backcrossed with C57Bl/6 inbred mice through ten successive generations and repeated intercrosses of $\mathrm{Fgl2} /$ fibrolenkin $^{+/}$, we again detected loss of Fgl2/fibrolenkin ${ }^{-1}$ homozygotes. At weaning we detected 62 and 14 Fgl2/fibroleukin ${ }^{+/-}$and $\mathrm{Fgl} /$ fibrolenkin $^{-/}$mice, respectively (observed ratio 1:0.2 versus expected 1:0.5 ratio; $\chi^{2}=7.6, P<0.01$ ).

Standard hematological profiles (hemoglobin, white blood cell count, and platelet counts), bleeding times, and prothrombin and partial thromboplastin time estimates in Fgl2/fibroleukin-deficient adult mice were similar to littermate controls (data not shown). Adult Fgl2/fibroleukin ${ }^{-/-}$mice were unremarkable compared with littermates with respect to gross anatomical examination, bleeding episodes, or longevity (2.5 years observation).

Procoagulant activity and Fgl2/fibrolenkin expression. Murine peritoneal macrophages infected in vitro with MHV-3 express a unique cell-surface calcium- and phospholipid-dependent procoagulant with direct prothrombinase activity. Cloning and heterologous expression of the MHV-3-induced procoagulant identified Fgl2/fibroleukin $(6,8,10)$. Strain-dependent susceptibility to MHV-3-induced fulminant hepatic failure correlates with the ability of Th1-type lymphocytes to induce expression of Fgl2/fibroleukin (22). Whereas the $\mathrm{A} / \mathrm{J}$ strain of mice is resistant to fulminant hepatic failure, the C57Bl/6 and CV129 strains are fully susceptible to MHV-3 and die within 4-7 days (23). Importantly, MHV-3 infection failed to induce the Fgl2/fibroleukin mRNA (Figure 1d) or procoagulant activity (Table 1) in thioglycollate-elicited peritoneal macrophages isolated from Fgl2/fibrolenkin ${ }^{-/-}$mice. LPS is known to induce $\mathrm{TF} /$ factor VII-dependent procoagulant activity in peritoneal macrophages. In contrast to the abrogation of MHV-3-induced procoagulant activity, the response to LPS was unaffected in Fgl2/fibrolenkin ${ }^{-/-}$cells (Table 1).
Susceptibility to MHV-3 infection and LPS exposure in Fgl2/fibroleukin ${ }^{-/}$mice. Mortality in MHV-3-infected (1,000 PFU) Fgl2/fibroleukin $/-$ mice was significantly blunted compared with Fgl2/fibrolenkin ${ }^{+/+}$littermates (Figure 3a), with heterozygotes showing an intermediate phenotype. Infection of $\mathrm{A} / \mathrm{J}$ and $\mathrm{C} 57 \mathrm{Bl} / 6$ mice recapitulated the resistant and susceptible phenotypes reported previously (16). Resistance of Fgl2/fibrolenkin /- mice to MHV-3-induced death was reproducible in multiple independent infections and across different preparations of viral stocks. We assessed the effects of strain background on MHV-3induced liver necrosis and survival in mice infected with MHV-3. Consistent with findings in the mixed $129 \mathrm{~Sv} / \mathrm{J} \times \mathrm{C} 57 \mathrm{Bl} / 6$ genetic background, findings indicated that $\mathrm{Fgl} / 2 / \mathrm{fibrolenkin}^{-/-}$mice on the $\mathrm{C} 57 \mathrm{Bl} / 6$ background were also resistant to MHV-3-induced liver necrosis and death (data not shown). The response of $\mathrm{Fgl}_{2} / \mathrm{fibrolenkin}^{-/}$mice to systemic inflammation induced by acute exposure to LPS was defined and compared with the LPS-hyporesponsive $\mathrm{C} 3 \mathrm{H} / \mathrm{HeJ}$ strain. All mouse strains, except for the $\mathrm{C} 3 \mathrm{H} / \mathrm{HeJ}$, exhibited signs of illness (mottled fur, inactivity, lethargy). The Fgl2/fibroleukin genotype

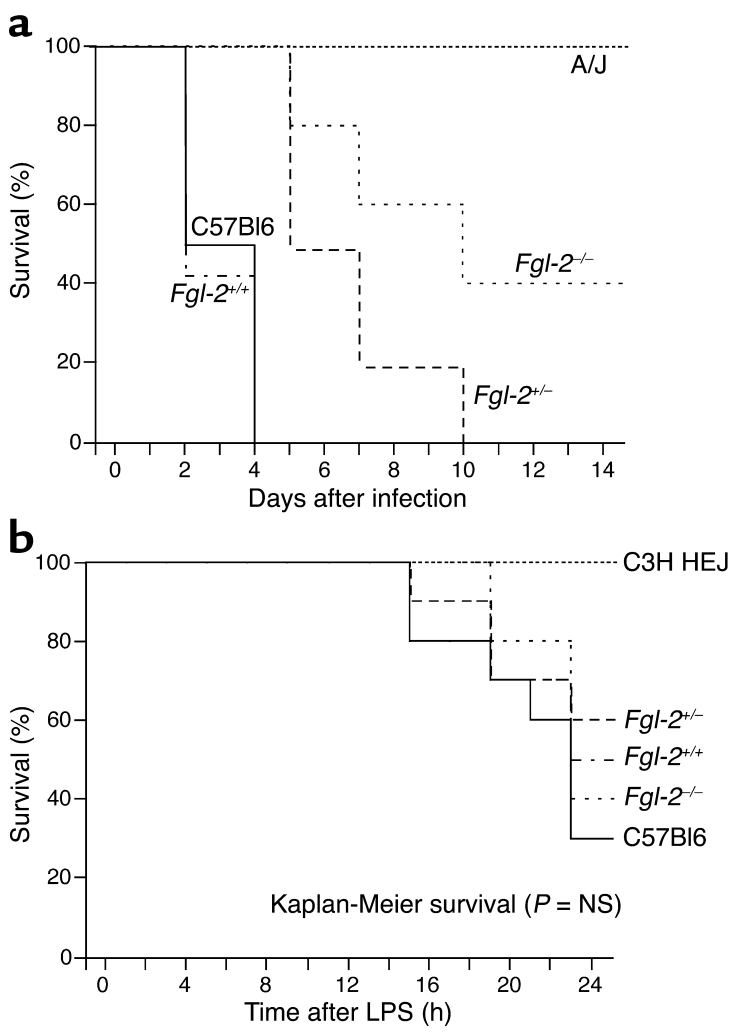

Figure 3

Fgl2/fibroleukin-deficient mice. (a) Mortality in MHV-3-infected (1,000 PFU) Fg/2/fibroleukin ${ }^{-1-}$ mice was significantly blunted compared with $\mathrm{Fg} / 2 /$ fibroleukin $^{+/+}$littermates, with heterozygotes showing an intermediate phenotype ( $n=10$ per group). C57Bl/ 6 and $\mathrm{A} / \mathrm{J}$ strains represent susceptible and resistant strains, as reported previously. (b) Mortality is not affected in LPS-treated Fgl2/fibroleukin-deficient mice. The $\mathrm{C} 3 \mathrm{H} / \mathrm{HeJ}$ strain is known to be LPS hyporesponsive. 

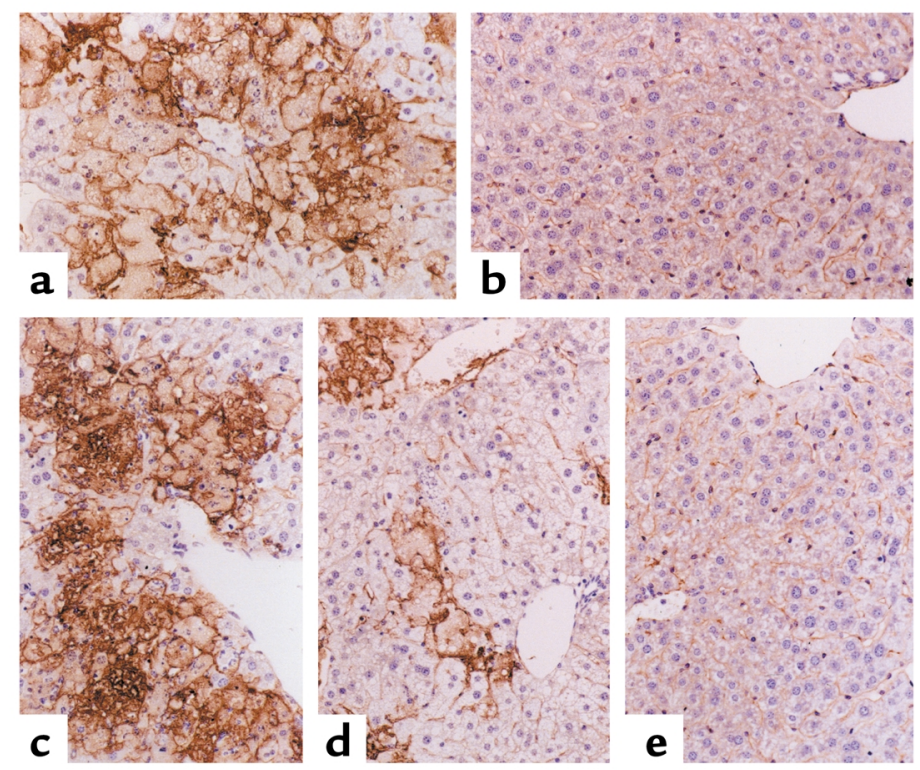

\section{Figure 4}

Fibrin deposition and cellular necrosis in livers of Fgl2/ fibroleukin-deficient mice following MHV-3 infection. (a) Liver from $\mathrm{C} 57 \mathrm{Bl} / 6$ (susceptible) mouse 3 days after infection. We note widespread confluent fibrin deposition and necrosis involving greater than $90 \%$ of liver tissue. (b) Liver from A/J (resistant) mouse. Liver tissue is normal with no fibrin deposition or necrosis. (c) Liver from Fg/2/fibroleukin ${ }^{+/+}$mouse. We noted that the liver shows confluent fibrin deposition and necrosis. (d) Liver from Fg/2/fibroleukin ${ }^{+/}$mouse. Fibrin deposition and necrosis and scattered foci are seen. (e) Liver from $\mathrm{Fg} / 2 /$ fibroleukin $^{-/-}$mouse. We noted that the livers were essentially free of fibrin deposition and necrosis. Only small, rare foci of fibrin deposition and necrosis were evident. failed to modify this response. Also, there was no difference in survival between non- $\mathrm{C} 3 \mathrm{H} / \mathrm{HeJ}$ strains as seen by Kaplan Meier analysis, demonstrating that disruption of the $f g l 2$ gene does not affect systemic LPS responses (Figure $3 b$ ).

When we assessed fibrin deposition in the liver and quantified hepatocellular necrosis in the viral hepatitis model (Figure 4), we observed effects of MHV-3 infection that were consistent with the phenotypic effect of the Fgl2/fibroleukin genotype on overall mortality, namely markedly decreased fibrin deposition and liver cell necrosis in Fgl2/fibrolenkin ${ }^{-/-}$mice. Marked elevations in serum ALT levels, a biochemical marker of liver cell histopathology (baseline values in normal mice are less than $50 \mathrm{IU} / \mathrm{l}$ ), were seen 3 days after viral infection in $\mathrm{C} 57 \mathrm{Bl} / 6$ and $\mathrm{Fgl}_{2} /$ fibrolenkin $^{+/+}$mice $(7,420 \mathrm{IU} / \mathrm{l}$ and $6,490 \mathrm{IU} / \mathrm{l})$. This can be contrasted with the trivial injury observed in A/J and Fgl2/fibrolenkin ${ }^{-/}$mice (54 IU/l and $87 \mathrm{IU} / \mathrm{l})$ or the intermediate response observed in Fgl2/fibroleukin $^{+/-}$mice (870 IU/l). When assessed at 14 days, modest elevations in ALT levels were evident in surviving Fgl2/fibrolenkin ${ }^{-1-}$ mice compared with the resistant A/J strain (625 versus $80 \mathrm{IU} / 1, P<0.05$ ). MHV-3 viral loads in the livers of A/J, Fgl2/fibrolenkin ${ }^{-1}, \mathrm{Fgl} /$ fibrolenkin $^{+/}$, and C57Bl/ 6 mice 3 days after infection were 8.0, 9.0, 8.2, and $9.0 \times 10^{7} \mathrm{PFU} / \mathrm{g}$ of tissue, indicating that the Fgl2/fibroleukin genotype did not have a major effect on the production of infectious virus in the liver. Fourteen days after infection, MHV-3 viral loads were significantly higher in surviving Fgl2/fibrolenkin ${ }^{-/-}$ mice compared with the resistant A/J strain $\left(6.0 \times 10^{9}\right.$ versus $2.0 \times 10^{2} \mathrm{PFU} / \mathrm{g}$ liver tissue). This indicates that although $\mathrm{Fgl}_{2} /$ fibrolenkin $^{-1}$ mice evidenced an important degree of protection against MHV-3-induced fulminant hepatic failure, in contrast to the resistant $\mathrm{A} / \mathrm{J}$ strain, they failed to eliminate the virus.

Increased Fgl2/fibrolenkin mRNA and protein in chronic active hepatitis $B$. To address the relevance of Fgl2/fibroleukin in human viral hepatitis, we studied patients with chronic hepatitis B. We have reported previously the coexpression of Fgl2/fibroleukin mRNA transcripts and fibrin in the livers of three patients with acute fulminant hepatic failure due to hepatitis B virus, an uncommon but devastating illness (7). In contrast, chronic hepatitis B virus infection is a common disease, with worldwide distribution. This serious clinical problem has protean adverse sequelae, including hepatic cirrhosis and hepatocellular carcinoma. Hepatitis B virus infection is especially endemic in Asia. Twenty-three patients with biochemical, histological, and clinical evidence of marked chronic viral hepatitis $B$ and 13 patients with minimal chronic viral hepatitis B were studied for hepatic Fgl2/ fibroleukin expression. Patient characteristics are noted in Table 2. Of note, $\mathrm{HBsAg}, \mathrm{HBeAg}$, anti-HBcAg, and

\section{Table 2}

Characteristics of the patients

$\begin{array}{lcc}\text { Characteristic } & \begin{array}{c}\text { Minimal chronic } \\ \text { hepatitis B } \\ (n=13)\end{array} & \begin{array}{c}\text { Marked chronic } \\ \text { hepatitis B } \\ (n=23)\end{array} \\ \text { Age (year) } & 43.8 & 36.0 \\ \quad \text { Mean } & 5.6 & 7.8 \\ \quad \text { SD } & 85 & 87 \\ \text { Male sex (\%) } & 100 & 100 \\ \text { HBsAg (\%) } & 46 & 30 \\ \text { HBeAg }(\%) & 85 & 91 \\ \text { Anti-HBcAg }(\%) & 54 & 39 \\ \text { HBV DNA } & & \\ \text { Serum ALT }(I U / I) & 300.5 & 586.0 \\ \text { Mean } & 238.9 & 404.2 \\ \text { SD } & & 452.1 \\ \text { Serum bilirubin }(\mu \mathrm{mol} / \mathrm{I})^{\mathrm{B}} & 78.3 & 191.7 \\ \text { Mean } & 89.4 & \\ \text { SD } & \end{array}$

AThe limit of detection of this assay was approximately ten genomes. ${ }^{\text {BTo }}$ convert values for bilirubin to $\mathrm{mg} / \mathrm{dl}$, divide by 17.1 . Hepatitis $B$ virus, $\mathrm{HBV}$. 
hepatitis B viral DNA load did not correlate with expression of $\mathrm{Fgl} 2 /$ fibroleukin or fibrin deposition. Although ALT and total bilirubin levels tended to be higher in patients with marked chronic hepatitis versus minimal chronic hepatitis B, there was considerable overlap in patient values. Twenty-one of 23 patients with marked chronic viral hepatitis $B$ were positive for Fgl2/fibroleukin expression, both of which were confirmed by in situ hybridization and immunohistochemistry. Fgl2/fibroleukin transcripts and immunoreactive protein were seen in macrophages and endothelial cells in areas of hepatic inflammation and necrosis, both in portal areas and in the sinusoids (Figure 5). Associated with the Fgl2/fibroleukin expression was fibrin deposition, as shown in Figure 5. Of the 13 patients with minimal chronic viral hepatitis B (Table 2), one patient demonstrated $\mathrm{Fgl}$ /2fibroleukin and fibrin expression. CD68-positive cells (Kupffer/macrophages) were clearly evident in all patients.

\section{Discussion}

Chronic infection with hepatitis B virus affects an estimated 300 million people on a global basis $(24,25)$. An estimated 1.25 million people in the United States are chronic carriers, defined as positive for $\mathrm{HBsAg}$ for more than 6 months (26). Although most carriers do not develop hepatic complications from chronic hepatitis B, $15-40 \%$ will develop serious sequelae during their lifetime, which is why a greater understanding is needed of the pathogenesis of viralinduced hepatocellular injury. We chose to study chronic hepatitis B viral infection in the AsianPacific region, where chronic infection is especially prevalent and patients usually acquire the infection at the time of birth or early in childhood. Some of these patients, whose infection originated earlier in life, demonstrate marked chronic hepatitis in the third and fourth decades of life. This disease is characterized by recurrent flares of hepatocellular injury. Minimal chronic viral hepatitis B may reflect either clinical remission of the latter or a more benign disease. Biopsy is necessary to distinguish the two disorders and has a direct influence on therapeutic choices. Flares of marked chronic hepatitis are postulated to reflect $\mathrm{T}$ lymphocyte-mediated immune responses to the virus (27). Indeed, T cell-mediated immune responses also play a prominent role in other forms of hepatocellular injury - autoimmune hepatitis, hepatotoxins, and concanavalin A hepatitis (28). It is not clear what explains why patients present with marked chronic hepatitis versus minimal chronic viral hepatitis B or what mediates transition from one form to the other. Intrahepatic production of IFN- $\gamma$ figures prominently in how viral hepatitis $\mathrm{B}$-specific $\mathrm{T}$ cells mediate hepatocellular injury in marked chronic hepatitis $(1,29,30)$.

The current study demonstrates that expression of $\mathrm{Fgl} 2 /$ fibroleukin mRNA and protein varies markedly in patients with marked chronic hepatitis B versus those with minimal chronic viral hepatitis $B$. Expression of Fgl2/fibroleukin is highly correlated with fibrin expression. We have reported previously expression of Fgl2/fibroleukin in three patients with hepatitis B-mediated fulminant hepatic failure, a devastating but rare complication of hepatitis B (7). Taken together with results reported here for marked chronic hepatitis, pharmacologic blockade of Fgl2/fibroleukin may offer an important new treatment approach in hepatitis viral B-induced disease. It is important to stress that these findings identify that an association exists between expression of Fgl2/fibroleukin, a prothrombinase, and the fibrin deposition that attends disease in marked chronic hepatitis B. Whether a cause and effect relationship exists between $\mathrm{Fgl} 2$ /fibroleukin expression and fibrin deposition requires future studies, such as clinical trials in patients with a specific inhibitor of Fgl2/fibroleukin. Future studies will also need to address how established therapies for hepatitis viral B-induced disease modulate Fgl2/fibroleukin expression, such as the effects of IFN- $\alpha$.

To begin to define a cause and effect relationship between the Fgl2/fibroleukin prothrombinase and
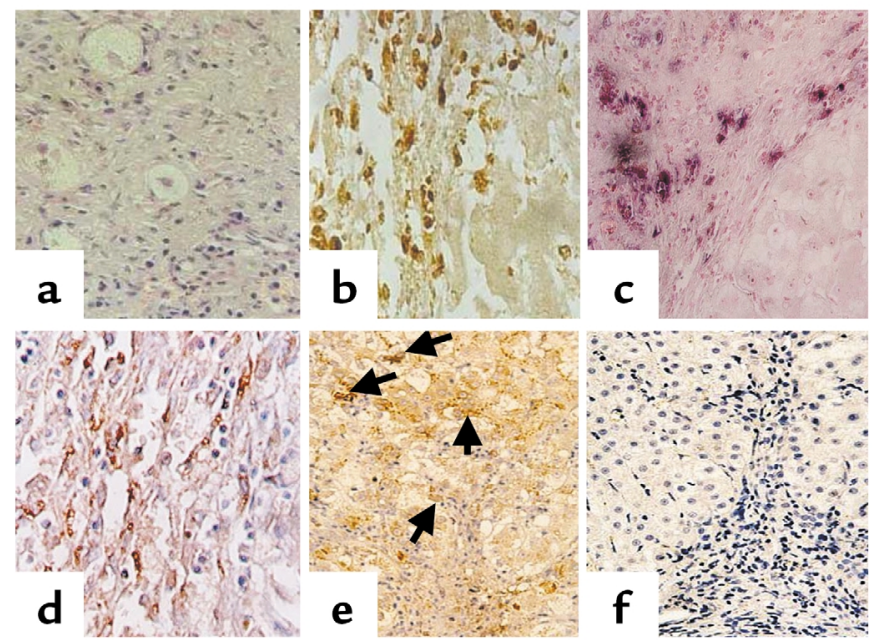

\section{Figure 5}

Fgl2/fibroleukin expression and fibrin deposition in human viral hepatitis B. Liver biopsy from a patient with marked chronic hepatitis B. (a) Area of necrosis and collapse of parenchyma is shown. The few remaining hepatocytes in this field show ballooning degeneration. Hematoxylin and eosin stain. $\times 400$. (b) Area of necrosis and collapse on left. Many macrophages are present in the area of collapse as shown by the positive CD68 immunoperoxidase stain. $\times 400$. (c) Area similar to that in $\mathbf{b}$, showing positivity of Fgl2/fibroleukin by in situ hybridization in the same distribution of the CD68-positive macrophages. $\times 200$. (d) Another area of acute necrosis and collapse showing positivity using Fgl2/fibroleukin Ab and immunoperoxidase stain. $\times 400$. (e) Similar area of necrosis to that in $\mathbf{d}$. Fibrin deposits by immunoperoxidase staining are shown by arrows. $\times 400$. (f) Patient with minimal chronic hepatitis $B$. This micrograph is representative and shows no evidence of hepatocellular necrosis, but a mixed mild inflammatory infiltrate confined to the portal and septal connective tissue. There is no evidence of Fgl2/fibroleukin in the parenchyma or in the areas of fibrosis areas by immunoperoxidase staining for Fgl2/fibroleukin. $\times 200$. 
hepatocellular injury in viral hepatitis we developed $\mathrm{Fgl}^{2} / \mathrm{fibroleukin}^{+/-}$and Fgl2/fibrolenkin ${ }^{-/-}$mice and examined the effect of Fgl2/fibroleukin genotype on in vitro and in vivo responses to MHV-3. Macrophages from Fgl2/fibrolenkin ${ }^{+/+}$mice responded to the MHV-3 virus with a robust procoagulant response. In contrast, macrophages from $\mathrm{Fgl2} /$ fibroleukin $^{-/}$mice generated no increase in procoagulant above control levels. LPS induced a similar procoagulant response in both. LPS is known to induce TF/factor VII-dependent procoagulant activity in peritoneal macrophages. In the presence of calcium, TF/factor VIIa activates the conversion of the zymogen precursor factor $\mathrm{X}$ into factor $\mathrm{Xa}$, the classical activator of thrombin generation. Using purified recombinant $\mathrm{Fgl}$ /fibroleukin prothrombinase generated in a baculovirus system and reconstituted into phospholipid-containing vesicles and MHV-3-infected murine peritoneal macrophages, we recently assessed rates of thrombin generation using one-stage clotting assays, ${ }^{125}$ I-labeled prothrombin cleavage assays, and a chromogenic substrate of thrombin, namely H-D-hexahydrotyrosyl-L-alanyl-L-arginine-p-nitroanalide-diacetate. Like factor Xa, the Fgl2/fibroleukin prothrombinase is dependent on calcium, phospholipids, and factor Va for full activity (10). Fgl2/fibroleukin prothrombinase differs from factor Xa in that activity on cell surfaces of MHV-3-infected peritoneal macrophages is resistant to extensive washing, consistent with the predicted transmembrane structure of the Fgl2/fibroleukin prothrombinase. Activity is not inhibited by antithrombin III and is not dependent on factor VII. The Fgl2/fibroleukin prothrombinase generates a unique thrombin cleavage fragment of approximately $24 \mathrm{kDa}(10)$. Neutralizing Ab's directed against Fgl2/fibroleukin inhibit MHV-3induced changes in the one-stage clotting assay (31). Such Ab's are specific in that they do not react with purified MHV-3- or LPS-induced procoagulants.

Mortality in MHV-3-infected Fgl2/fibrolenkin ${ }^{-/}$mice was significantly reduced compared with Fgl2/fibro$\mathrm{leukin}^{++}$littermates; therefore, infection of $\mathrm{Fgl} /$ fibrolenkin $^{-/-}$and Fgl2/fibrolenkin ${ }^{+/+}$mice tended to recapitulate the resistant and susceptible phenotypes reported previously for $\mathrm{C} 57 \mathrm{Bl} / 6$ and $\mathrm{A} / \mathrm{J}$ inbred strains, respectively (16). Also, targeted ablation of the Fgl2/fibroleukin gene and neutralizing Ab's against the Fgl2/fibroleukin prothrombinase resulted in comparable effects on MHV-3 pathogenesis (23). The response to LPS was not modified by Fgl2/fibroleukin genotype and contrasted with the effect in the LPShyporesponsive $\mathrm{C} 3 \mathrm{H} / \mathrm{HeJ}$ strain. When we assessed fibrin deposition in the liver and quantified hepatocellular necrosis, we observed effects of MHV-3 infection that were consistent with the effect of the Fgl2/fibroleukin genotype on overall mortality, namely, markedly decreased fibrin deposition and hepatocellular injury in Fgl2/fibrolenkin ${ }^{-/-}$mice. Previous studies have suggested the existence of multiple genetic modifiers for the MHV-3-induced fulminant hepatitis phenotype (19), including the CEACAM1 MHV viral receptor glycoprotein (32). MHV-3 viral loads in the livers did not vary with the Fgl2/fibroleukin genotype. This contrasts with the effects of targeted disruption of the CEACAM1 MHV viral receptor, which modifies hepatotrophic host-viral interactions and decreases viral loads in the livers of MHV-3-infected mice (33). We did not detect an important effect of ablating the Fgl2/fibroleukin gene on MHV-3 viral loads in the liver. To the extent that MHV-3 viral hepatitis can be used to model viral hepatitis B in humans, these cumulative findings can be taken to indicate that hepatocellular injury induced by MHV- 3 is critically dependent on the Fgl2/fibroleukin prothrombinase.

What are the other biological roles for Fgl2/fibroleukin? When generating the Fgl2/fibroleukin-deficient murine model, we identified loss of Fgl2/fibrolenkin ${ }^{-1}$ mice on both a mixed $129 \mathrm{~Sv} / \mathrm{J} \times \mathrm{C} 57 \mathrm{Bl} / 6$ genetic background and C57Bl/ 6 inbred background. Coagulation and procoagulant factor deficiencies are known to result in early or "occult" pregnancy failure due to perturbations at the fetal-maternal interface and can be maternal $(34,35)$ or fetal in origin $(36-39)$. We have reported previously constitutive $\mathrm{Fgl} 2$ /fibroleukin prothrombinase mRNA and protein expression on murine trophectoderm (17). Future studies will be necessary to determine whether reconstitution of $\mathrm{Fgl} 2 /$ fibroleukin expression in extraembryonic tissues rescues $\mathrm{Fgl}_{2} / \mathrm{fibrolenkin}^{-/-}$mice from early lethality or whether loss of $\mathrm{Fgl}_{2} /$ fibrolen kin $^{-/-}$ mice reflects other important functional contributions of Fgl2/fibroleukin during development. When studying adult $\mathrm{Fgl} 2 /$ fibroleukin-deficient mice, we failed to identify baseline alterations in standard hematological and coagulation profiles or bleeding times. Although adult $\mathrm{Fgl} /$ fibrolenkin ${ }^{-/-}$mice were unremarkable compared with littermates with respect to bleeding episodes or longevity, we anticipate more detailed future analysis of the coagulation cascade.

This Fgl2/fibroleukin prothrombinase bypasses the $\mathrm{TF} /$ factor VII extrinsic pathway and directly converts prothrombin to thrombin. Stimuli known to induce expression of the Fgl2/fibroleukin mRNA and protein (e.g, IFN- $\gamma$ treatment [see ref. 13]), MHV-3 viral infection, xenotransplantation) are distinct from those that elicit $\mathrm{TF} /$ factor VII-dependent fibrin generation (e.g., LPS). Future studies will need to define the disease settings in which Fgl2/fibroleukin expression directs local fibrin generation. It is of interest that a coronavirus can induce tissue-specific expression of the Fgl2/fibroleukin prothrombinase. Using a set of parental and recombinant MHV-3 strains, we mapped the viral genetic determinants that induce fulminant hepatitis in susceptible murine strains (40). These studies identified a critical role for the MHV-3 coronavirus nucleocapsid gene product in the transcriptional activation of the gene. Whether coronavirus infection in other species also induces Fgl2/fibroleukin prothrombinase expression remains to be determined. In this respect it will be important to define whether expression of the Fgl2/fibroleukin prothrombinase contributes to pulmonary parenchymal 
injury in human patients with severe acute respiratory syndrome (SARS), given that coronavirus infection contributes to this newly recognized illness (41-44).

Our results provide compelling evidence for a role of the Fgl2/fibroleukin prothrombinase in viral hepatitis. Collectively, these data argue that the Fgl2/fibroleukin prothrombinase is a logical target for molecular manipulation and offers the hope for the development of new treatment approaches for patients with fulminant and marked chronic viral hepatitis.

\section{Acknowledgments}

This work was supported by operating grants from the Canadian Institutes of Health Research (MOP 37778 to P.A. Marsden; MOP 37777 to G.A. Levy and D.A. Clark). Q. Ning is supported by the Natural Science Foundation of China (NSFC30170846) and is an Outstanding Young Investigator (NSFC30225040). Mouse genotyping was kindly performed by the Mouse Genetic Mapping Facility of the Toronto Centre for Modeling Human Disease. P.A. Marsden is a Heart and Stroke Foundation of Canada Career Investigator.

1. Guidotti, L.G., and Chisari, F.V. 1996. To kill or to cure: options in host defense against viral infection. Curr. Opin. Immunol. 8:478-483.

2. Levy, G.A., MacPhee, P.J., Fung, L.S., Fisher, M.M., and Rappaport, A.M. 1983. The effect of mouse hepatitis virus infection on the microcirculation of the liver. Hepatology. 3:964-973.

3. Mackman, N. 1997. Regulation of the tissue factor gene. Thromb. Haemost. 78:747-754.

4. Osterud, B., and Bjorklid, E. 2001. The tissue factor pathway in disseminated intravascular coagulation. Semin. Thromb. Hemost. 27:605-617.

5. Levy, G.A., Leibowitz, J.L., and Edgington, T.S. 1981. Induction of monocyte procoagulant activity by murine hepatitis virus type 3 parallels disease susceptibility in mice. J. Exp. Med. 154:1150-1163.

6. Parr, R.L., et al. 1995. Association of mouse fibrinogen-like protein with murine hepatitis virus-induced prothrombinase activity. J. Virol. 69:5033-5038.

7. Levy, G.A., et al. 2000. Molecular and functional analysis of the human prothrombinase gene (HFGL2) and its role in viral hepatitis. Am. J. Pathol. 156:1217-1225.

8. Yuwaraj, S., Ding, J., Liu, M., Marsden, P.A., and Levy, G.A. 2001. Genomic characterization, localization, and functional expression of FGL2, the human gene encoding fibroleukin: a novel human procoagulant. Genomics. 71:330-338.

9. Ruegg, C., and Pytela, R. 1995. Sequence of a human transcript expressed in T-lymphocytes and encoding a fibrinogen-like protein. Gene. 160:257-262.

10. Chan, C.W., et al. 2002. Kinetic analysis of a unique direct prothrombinase, fgl2, and identification of a serine residue critical for the prothrombinase activity. J. Immunol. 168:5170-5177.

11. Koyama, T., Hall, L.R., Haser, W.G., Tonegawa, S., and Saito, H. 1987. Structure of a cytotoxic T-lymphocyte-specific gene shows a strong homology to fibrinogen beta and gamma chains. Proc. Natl. Acad. Sci. U. S. A. 84:1609-1613.

12. Qureshi, S., et al. 1995. Mouse hepatitis virus-3 induced prothrombinase (Fgl2) maps to proximal chromosome 5. Genomics. 29:307-309.

13. Lafuse, W.P., Castle, L., Brown, D., and Zwilling, B.S. 1995. The cytotoxic T lymphocyte gene FIBLP with homology to fibrinogen beta and gamma subunits is also induced in mouse macrophages by IFN-gamma. Cell Immunol. 163:187-190.

14. Marazzi, S., et al. 1998. Characterization of human fibroleukin, a fibrinogen-like protein secreted by T lymphocytes. J. Immunol. 161:138-147.

15. Ding, J.W., et al. 1997. Fulminant hepatic failure in murine hepatitis virus strain 3 infection: tissue-specific expression of a novel fgl2 prothrombinase. J. Virol. 71:9223-9230.

16. MacPhee, P.J., Dindzans, V.J., Fung, L.S., and Levy, G.A. 1985. Acute and chronic changes in the microcirculation of the liver in inbred strains of mice following infection with mouse hepatitis virus type 3. Hepatology. 5:649-660.

17. Knackstedt, M., et al. 2001. Activation of the novel prothrombinase, fg12, as a basis for the pregnancy complications spontaneous abortion and pre-eclampsia. Am. J. Reprod. Immunol. 46:196-210.

18. Law, D.A., et al. 1999. Integrin cytoplasmic tyrosine motif is required for outside-in alphaIIbbeta3 signaling and platelet function. Nature. 401:808-811.

19. Dindzans, V.J., Skamene, E., and Levy, G.A. 1986. Susceptibility/resistance to mouse hepatitis virus strain 3 and macrophage procoagulant activity are genetically linked and controlled by two non-H-2-linked genes. J. Immunol. 137:2355-2360.

20. Phillips, M.J., et al. 1992. Post-transplant recurrent hepatitis B viral liver disease. Viral-burden, steatoviral, and fibroviral hepatitis B. Am. J. Pathol. 140:1295-1308.

21. Lok, A.S., Heathcote, E.J., and Hoofnagle, J.H. 2001. Management of hepatitis B: 2000 - summary of a workshop. Gastroenterology. 120:1828-1853.

22. Liu, M.F., et al. 1998. Resistance of naive mice to murine hepatitis virus strain 3 requires development of a Th1, but not a Th2, response, whereas pre-existing antibody partially protects against primary infection. Adv. Exp. Med. Biol. 440:415-423.

23. Li, C., et al. 1992. Monoclonal antiprothrombinase (3D4.3) prevents mortality from murine hepatitis virus (MHV-3) infection. J. Exp. Med. 176:689-697.

24. Maupas, P., and Melnick, J.L. 1981. Hepatitis B infection and primary liver cancer. Prog. Med. Virol. 27:1-5.

25. Hoofnagle, J.H., and di Bisceglie, A.M. 1997. The treatment of chronic viral hepatitis. N. Engl. J. Med. 336:347-356.

26. Pawlotsky, J.M. 2002. Molecular diagnosis of viral hepatitis. Gastroenterology. 122:1554-1568.

27. Guidotti, L.G., and Chisari, F.V. 2001. Noncytolytic control of viral infections by the innate and adaptive immune response. Annu. Rev. Immunol. 19:65-91.

28. Hong, F., et al. 2002. Opposing roles of STAT1 and STAT3 in T cell-mediated hepatitis: regulation by SOCS. J. Clin. Invest. 110:1503-1513. doi: $10.1172 /$ JCI200215841.

29. Leifeld, L., et al. 2002. Imbalanced intrahepatic expression of interleukin 12 , interferon gamma, and interleukin 10 in fulminant hepatitis B. Hepatology. 36:1001-1008.

30. Kakimi, K., et al. 2001. Blocking chemokine responsive to gamma2 /interferon (IFN)-gamma inducible protein and monokine induced by IFN-gamma activity in vivo reduces the pathogenetic but not the antiviral potential of hepatitis B virus-specific cytotoxic T lymphocytes. J. Exp. Med. 194:1755-1766.

31. Fung, L.S., et al. 1991. Monoclonal antibody analysis of a unique macrophage procoagulant activity induced by murine hepatitis virus strain 3 infection. J. Biol. Chem. 266:1789-1795.

32. Ohtsuka, N., and Taguchi, F. 1997. Mouse susceptibility to mouse hepatitis virus infection is linked to viral receptor genotype. J. Virol. 71:8860-8863.

33. Blau, D.M., et al. 2001. Targeted disruption of the Ceacam1 (MHVR) gene leads to reduced susceptibility of mice to mouse hepatitis virus infection. J. Virol. 75:8173-8186.

34. Erlich, J., et al. 1999. Tissue factor is required for uterine hemostasis and maintenance of the placental labyrinth during gestation. Proc. Natl. Acad. Sci. U. S. A. 96:8138-8143.

35. Iwaki, T., et al. 2002. Fibrinogen stabilizes placental-maternal attachment during embryonic development in the mouse. Am. J. Pathol. 160:1021-1034.

36. Isermann, B., Hendrickson, S.B., Hutley, K., Wing, M., and Weiler, H. 2001. Tissue-restricted expression of thrombomodulin in the placenta rescues thrombomodulin-deficient mice from early lethality and reveals a secondary developmental block. Development. 128:827-838.

37. Sun, W.Y., et al. 1998. Prothrombin deficiency results in embryonic and neonatal lethality in mice. Proc. Natl. Acad. Sci. U. S. A. 95:7597-7602.

38. Cui, J., O'Shea, K.S., Purkayastha, A., Saunders, T.L., and Ginsburg, D. 1996. Fatal haemorrhage and incomplete block to embryogenesis in mice lacking coagulation factor V. Nature. 384:66-68.

39. Bugge, T.H., et al. 1996. Fatal embryonic bleeding events in mice lacking tissue factor, the cell-associated initiator of blood coagulation. Proc. Natl. Acad. Sci. U. S. A. 93:6258-6263.

40. Ning, Q., et al. 1999. The nucleocapsid protein of murine hepatitis virus type 3 induces transcription of the novel fgl2 prothrombinase gene. J. Biol. Chem. 274:9930-9936.

41. Poutanen, S.M., et al. 2003. Identification of Severe Acute Respiratory Syndrome in Canada. N. Engl. J. Med. 348:1995-2005.

42. Peiris, J.S.M., et al. 2003. Coronavirus as a possible cause of severe acute respiratory syndrome. Lancet. 361:1319-1325.

43. Ksiazek, T.G., et al. 2003. A novel Coronavirus associated with Severe Acute Respiratory Syndrome. N. Engl. J. Med. 348:1953-1966.

44. Drosten, C., et al. 2003. Identification of a novel Coronavirus in patients with Severe Acute Respiratory Syndrome. N. Engl. J. Med. 348:1967-1976. 